the methods of Hudson. Before using, all the sugars were dried, at $50^{\circ}$, to constant weight in vacuo over conc. sulfuric acid.

The hydrogen ion concentration was measured, by a combination of electrometric and colorimetric methods, as described by Nelson and Vosburgh. 'The determinations were made at the same temperature as that of the sugar solution studied. In order to do this, it was necessary first to measure the temperature coefficient of the saturated calomel cell. This work will be described in another paper by Fales and Beegle.

NEW YORE CITY.

\title{
RELATION BETWEEN INTENSITY OF TYNDALL BEAM AND SIZE OF PARTICLES. ${ }^{2}$
}

By Richard C. Tolman, Roscon H. Girke, Adin P. Brooks, Albert G. Herrman, ROBERT S. MULITKEN and Harry DeW. SMYth.

Received January $17,1919$.

\section{Introduction.}

In a previous article ${ }^{3}$ a simple Tyndallmeter has been described for measuring the strength of Tyndall beam in smokes and suspensions. The strength of the Tyndall beam has been shown to be directly proportional to the concentration of a smoke or of a suspension, ${ }^{4}$ and it is the purpose of this article to show the relation between the strength of Tyndall beam and the size of particles. Work along this line on suspensions of colloidal sulfur has already been performed by Mecklenburg." Mecklenburg's, results show that for particles in the range of size which he examines, the Tyndall beam became more intense the larger the particles, concentration remaining uniform. It has already been pointed out, however, in our article ${ }^{6}$ on "The Disappearance of Smoke in a Confined Space," that at a given concentration the intensity of Tyndall beam became larger with finer particles. For this reason a further investigation of the subject seemed necessary and a determination, if possible, of the exact relation between strength of Tyndall beam and size of particle. The general results of the work are to show that for the range of particles in actual smokes $\left(5 \times 10^{-6}\right.$ to $10^{-4} \mathrm{~cm}$.) and for particles in suspensions $10^{-4} \mathrm{~cm}$. in diameter up, the Tyndall beam becomes more intense at a given concentration the greater the sub division. Mecklenburg's results were all for particles below $10^{-4} \mathrm{~cm}$. Nevertheless at that size of particle his results

1 Terrs Journal, 39, 810 ( $19 \mathrm{r} 7$ ).

${ }^{2}$ The work described in this article was carried out by the Dispersoid Section, Research Division, Chemical Warfare Service and has been approved for publication by Major-General William L. Sibert, Director of Chemical Warfare Service, U. S. A.

3 "THIS JoURNAL, 4I, 297 (I9I9).

\& Ibid., 4I, 300 (1919).

' Kolloid-Z., 16, 97 (1915).

8 Turs JournaI, 4I, 304 (1919). 
still showed the Tyndall beam to be increasing with increased size of particle.

\section{Particles in Smokes.}

Method.-Owing to the great difficulty of obtaining a smoke with particles of one given size, no attempt to obtain exact quantitative data for the relation between Tyndallmeter reading and size of particle was made in the case of smokes. We were able, however, to obtain absolutely convincing qualitative information which showed that the Tyndallmeter reading at a given concentration increases with degree of subdivision. The method consisted essentially in setting up a smoke in an enciosed space and making a set of simultaneous measurements of its Tyndallmeter reading and its concentration. We find in every case that the Tyndallmeter reading fell off with the time more rapidly than the concentration, owing to the coalescing of the particles. Since we have already shown that for a given size of particle the Tyndallmeter reading is exactly proportional to concentration, this result is a definite proof that coalescence of the particles at a given concentration leads to a decreased Tyndallmeter reading.

Experimental Method.-The substance chosen for the production of smokes to be experimented on was rosin. This material was finely ground and the smokes were produced by volatilization and recondensation.

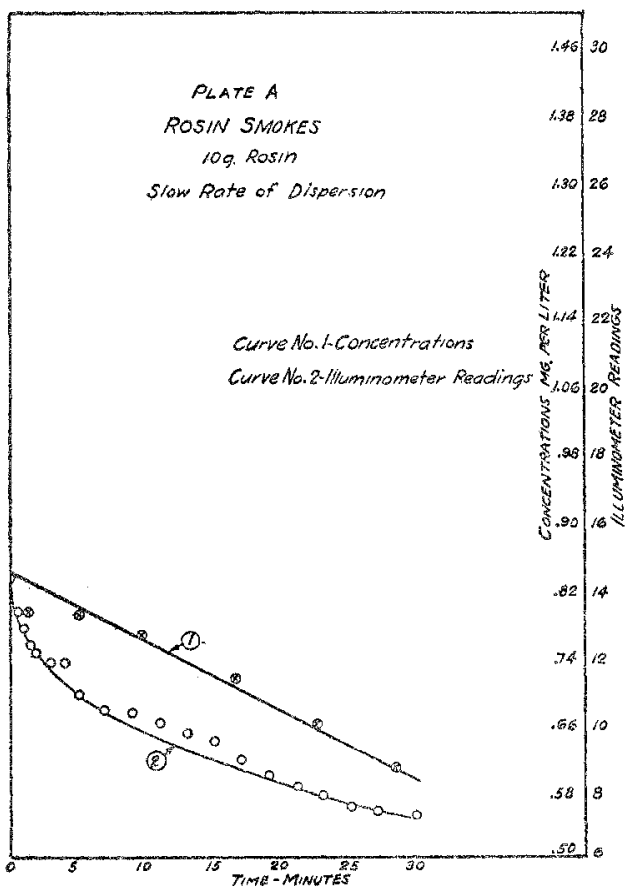

The method of smoke production was arranged so that, if desired, smaller or larger particles could be produced by varying the rate at which the volatilization took place.

The smokes were set up in a box having a capacity of Io cu. m. provided with a Sirocco blower which slowly circulated the smoke through the Tyndallmeter as already described in the article on "The Disappearance of Smokes." "Simultaneous with the Tyndallmeter determinations a sample of smoke was drawn out at the rate of 5 liters per minute through the Tyndallmeter connections and passed through an electrical analyzer such as has already been s Loc. cit. 
described, ${ }^{\mathrm{i}}$ the smoke was precipitated on the foil and the concentration determined by weighing. It was found by microscopic observation that the precipitate of rosin smoke was very uniform and that there were no large particles. In the case of non-uniform and impure smokes the irregular and accidental presence of a few large particles can very materially disturb the true results owing to their great weight.

Experimental Results. - The experimental results obtained in this work are shown in Plates $A, B$ and C. It will be seen from these plates that at the start of a run the Tyndallmeter reading always falls off more rapidly than the concentration. This is due to the fact that when freshly produced the smoke is coalescing and is definite proof that at a given concentration the Tyndallmeter reading increases with increased subdivision. In the results of the experiment shown on Plate $B$, the same amount of rosin was employed as for Plate $A$ but a higher rate of dispersion, leading to an original finer subdivision in particles. This accounts for the higher Tyndallmeter readings and the higher rate of dissipation obtained at the start of the run. This is, of course, additional proof that the increased subdivision means increased Tyndallmeter reading.

In the experiment shown on Plate $\mathrm{C}$ the same rate of smoke production was used as for Plate

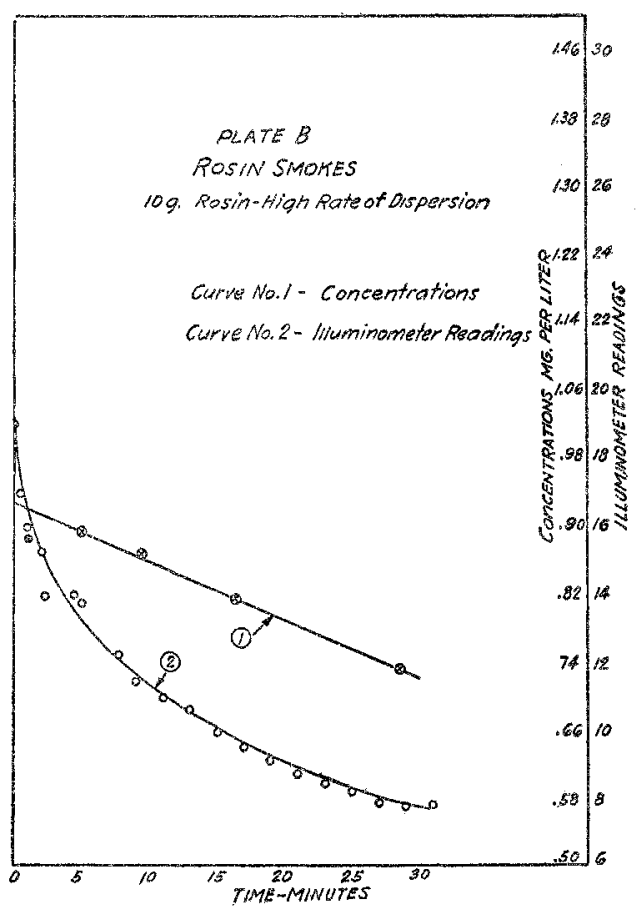

$B$, but a larger anount of rosin was employed, giving us a higher concentration of smoke. This leads, of course, to an increased rate, both for the decrease in concentration and for the decrease in Tyndallmeter reading as already shown in the article on "The Disappearance of Smokes in a Confined Space."

In order to show numerically the fact that the Tyndallmeter reading falls of more rapidly than concentration, we append the following table which gives a comparison for the three different experiments of the percentage drop in Tyndallmeter reading and the percentage drop in concentration at the end of $r_{5}$ and at the end of 30 minutes.

2 Thris Journal, following article. 
TABLE I.

15 minutes.

Plate.

Plate A......., , $4 \mathrm{I}, 4$

Plate B......... 47.3

Plate C.......... 46.0

\% drop
Tyndall.
41.4
47.3
46.0

\% drop
concentration.
I4.
II .7
I 2.7

30 minutes.

$\begin{array}{cc}\text { \% drop } & \begin{array}{c}\% \text { drop } \\ \text { Tyndati. }\end{array} \\ \begin{array}{cc}\text { concentration. } \\ 48.2\end{array} & 28.6 \\ 58.5 & 23.4 \\ 62.0 & 26.0\end{array}$

These plates are merely samples from a large number of runs which had been made on rosin and on other smoke-producing substances. In an enormous amount of work we have never found a single case in which

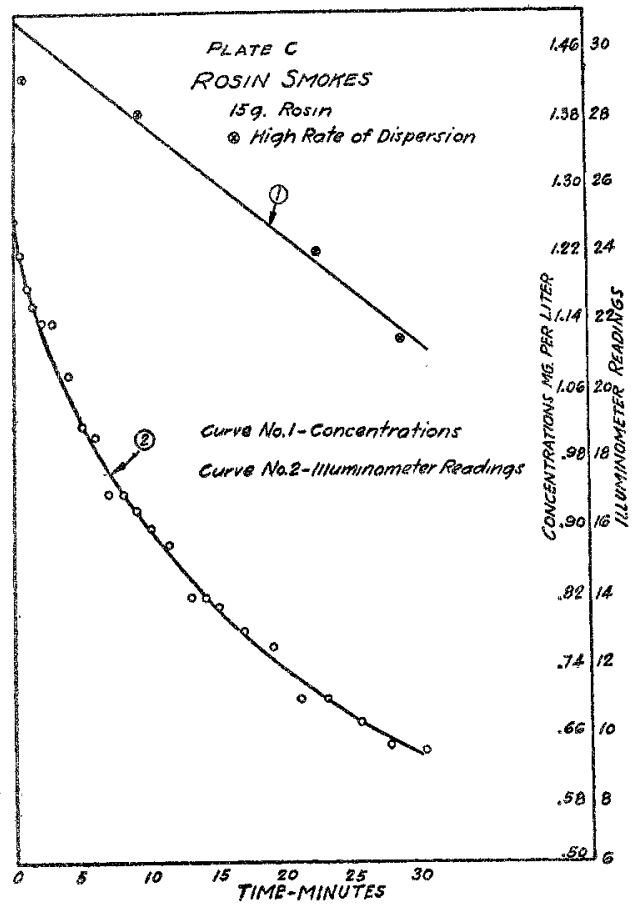
the rate at which the Tyndallmeter reading falls off is less than the rate at which the concentration falls off.

Actual Size of Smoke Particles.-As already stated, owing to the fact that the particles in an actual smoke are not all uniform in size, no attempt was made in these experiments actually to measure the size of particle present at any given instant. It is nevertheless important to note the general range of smoke particle size and this has been the subject of a number of investigations made in connection with this work. The methods used for such measurement are described in an article by Wells and Gerke, ${ }^{1}$ and these measurements

have shown that particles in such smokes as we have experimented on range in size from $5 \times 10^{-6} \mathrm{~cm}$. to $10^{-4} \mathrm{~cm}$. in diameter and these results have also shown, by taking successive samples, that the number of fine particles actually decreases with the time and the number of large particles increases.

Our results thus show that in the range of particle size from $10^{-6} \mathrm{~cm}$. to $10^{-4} \mathrm{~cm}$. Tyndallmeter reading increases with increased dispersion.

\section{Particles in Suspension.}

Method.-In the case of a suspension it is possible to prepare samples by fractionation, each having a definite uniform size of particle, and hence to obtain definite quantitative information as to the relation be-

1 Thus Jotrnat, 4r, 312 (1919). 
tween Tyndall beam and size of particle. Our work consisted in the preparation of five different suspensions of silica particles which varied in size from ro to $206 \times 10^{-5} \mathrm{~cm}$. The size of these particles was then determined by direct microscopic measurement, the concentration of the suspension determined by evaporation to dryness and a measurement made of the intensity of the Tyndall beam.

Preparation of the Suspensions.-Five different suspensions were prepared from ground silica by mixing the silica with distilled water and carrying out fractional settlings and centrifugations.

The largest particles $(206 \times$ $\mathrm{IO}^{-5} \mathrm{~cm}$.) were those which settled between the limits of I and ro minutes, the second

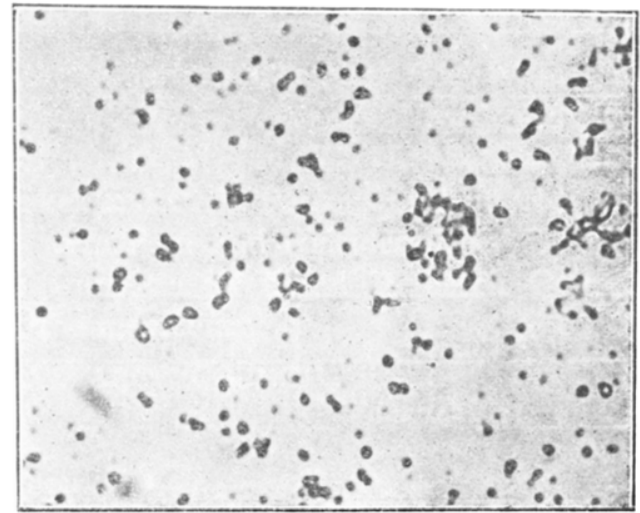

Fig. I. size of particles $\left(93.9 \times 10^{-5} \mathrm{~cm}\right.$.) were those which settled between the limits of Io and 15 minutes, the third size of particles $\left(62.4 \times \mathrm{ro}^{-5}\right.$ $\mathrm{cm}$.) were those which settled between the limits of 20 and 30 minutes, the fourth size of particles $\left(22.4 \times 10^{-5} \mathrm{~cm}\right.$.) were those which remained in suspension after 30 minutes but could be thrown out in 5 minutes of

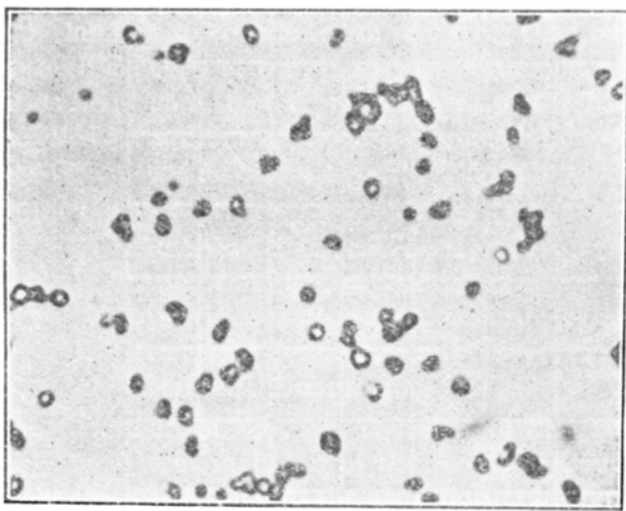

Fig. 2. centrifugation at a speed of I 400 R. P. M., using a Size 3 International Equipment Co. centrifuge. The finest particles $\left(9.97 \times \mathrm{ro}^{-5} \mathrm{~cm}\right.$.) were those which had not settled after 9 days' standing, but could be centrifuged out in 15 minutes at a speed of 2700 R. P. M. Each of these suspensions was allowed to settle for the time interval noted or was centrifuged a considerable number of times in order to secure uniformity of size.

Figs. I, 2, 3, 4 and 5 are made from photomicrographs of samples taken from the 5 suspensions. The success of the fractionation process will be evident from an examination of the prints. The magnification is about 950 diameters. 
Measurement of Size.-In order to determine the size of the particles in a given suspension, a few drops of the liquid were allowed to evaporate to dryness on a microscopic slide. The diameters of individual particles were then determined using a Spencer microscope with a $160 \mathrm{~mm}$. tube

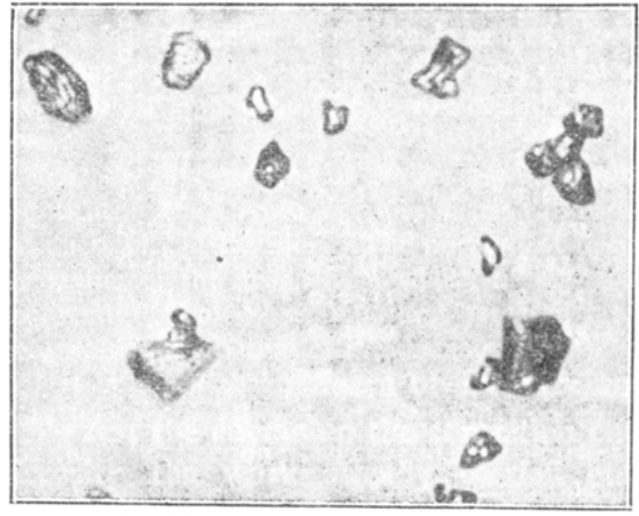

Fig. 3 . length and a $3 \mathrm{~mm}$. objective, and a Bausch and Lomb Filar micrometer which was calibrated directly against a Bausch and Lomb stage micrometer. About 50 or 60 separate particles were measured for each suspension, the maximum deviation from the mean diameter being only about $50 \%$, the average deviation being very much less.

Determination of Concentration.-The concentration of the suspensions used was determined by evaporating to dryness, in a platinum evaporating dish, 50 or Ioo cc. of the suspension. For each determination duplicate analyses were made, the dishes being dried to a constant weight. A correction was applied to the analyses for impurities in the distilled water. This was determined by evaporating Ioo cc. of distilled water to dryness and weighing the residue. This correction amounts to $0.0003 \mathrm{~g}$. per Ioo cc. of distilled water, while the lowest concentration used was $0.03 \mathrm{~g}$. Suspensions of lower concentration than the original sample were made by dilution.

Determination of the Intensity of the Tyndall Beam.-The Tyndallmeter

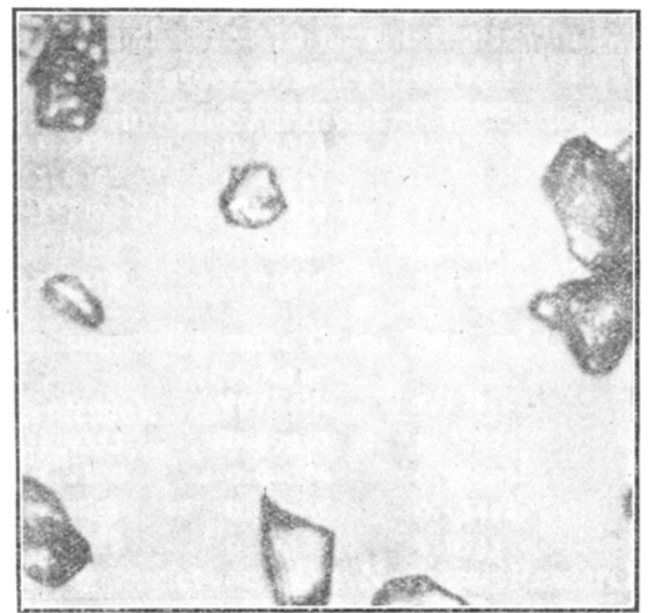

Fig. 4. readings were made with the Tyndallmeter, already described, by placing the suspension in a cylindrical glass tube and inserting into the examining chamber of the instrument. An average of ro separate read- 
ings was taken for each observation. In order to eliminate error, onehalf the readings were made by approaching the setting from one direction and the other half from the other direction. The maximum deviation in setting was in the neighborhood of $5 \%$ which is about the usual accuracy of the instrument.

The readings had to be corrected for the reflection of light from the glass container when filled with distilled water. This correction was 0.6 and hence became quite important at high dilution, and as a matter of fact limited the dilutions to which measurements could be carried out.

The coarser suspensions had to be read immediately after shaking, owing to the rapidity with which they settled out.

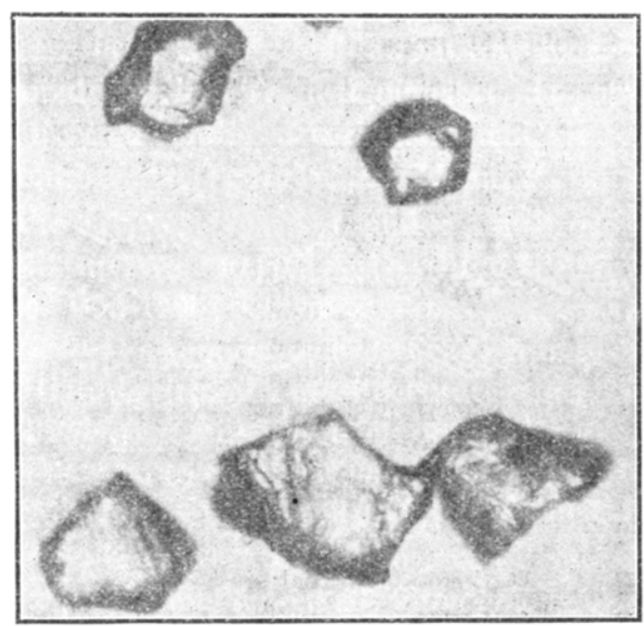

Fig. 5.

All the measurements were made in a dark room in order to preserve the sensitiveness of the reader's eye and to prevent extraneous light entering the tube.

Experimental Results. - It would take up too much space to present the actual figures for all the measurements made. In order to give an

TABLE II.

Diameter of Single Particles in Filar Micrometer Divisions.

$\begin{array}{rrrr}7 & \text { II } & 8 & \text { IO } \\ 9 & 5 & 8 & 7 \\ 9 & 5 & 7 & \text { IO } \\ 4 & 6 & 4 & 6 \\ 6 & 7 & 8 & 4 \\ 5 & 6 & 7 & 4 \\ 5 & 7 & 8 & 7 \\ 4 & 5 & 9 & 6 \\ 9 & 6 & \text { 10 } & \text { 10 } \\ 8 & 8 & 8 & 6 \\ 7 & 9 & 6 & 7 \\ 8 & 5 & 6 & 6 \\ 8 & 11 & 7 & 5 \\ 6 & 7 & 6 & 5 \\ \text { I5 } & 6 & 7 & 5 \\ 5 & 5 & 7 & 9 \\ \text { Average 7.12 micrometer divisions } & =9.97 \times \text { 10 }^{-5} \mathrm{~cm} .\end{array}$


idea, however, of the accuracy of the measurements, the actual measurements are given below for the suspension of the smallest size particles.

Table II gives the individual measurement of the diameter of the particles.

These measurements on the smallest size particles are of course less accurate than those on the largest particles which were 200 times as big.

Table III presents the concentration data and average Tyndallmeter readings for the particles of smallest diameter.

TABLE III.

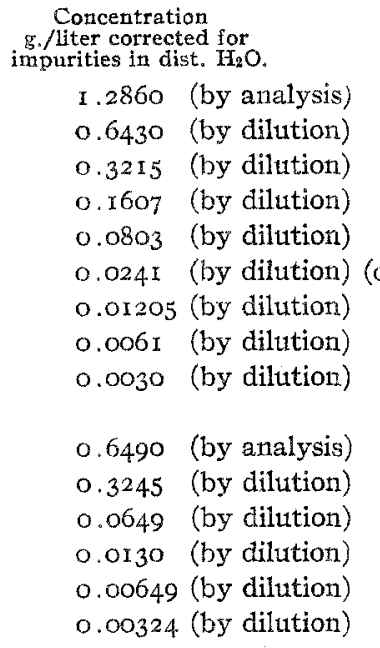

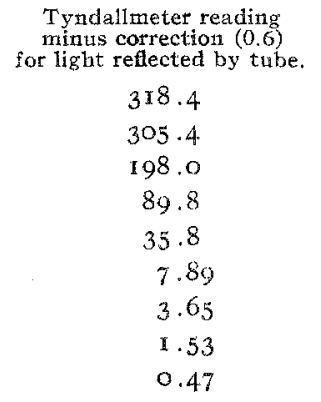

CHęcK Run.

$$
\begin{array}{r}
299.6 \\
180.4 \\
25.1 \\
3.55 \\
x .55 \\
0.49
\end{array}
$$

Plots.-Data for all the 5 suspensions are presented in the plots in Plates I, II, III, IV and V.

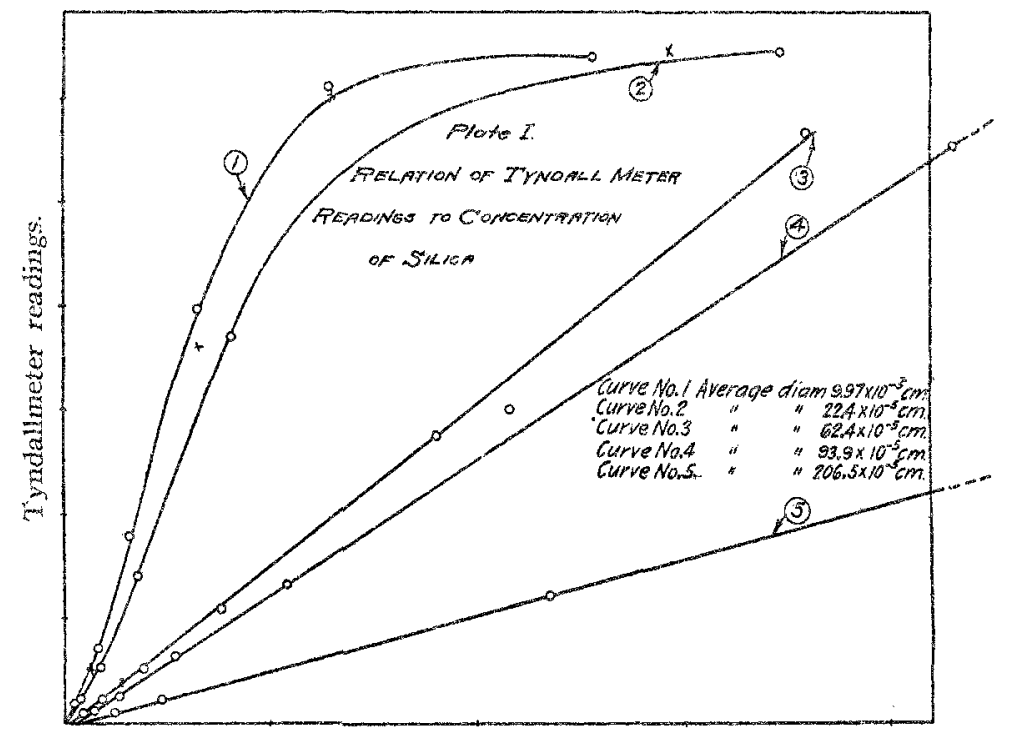

Concentration. 
Plate I shows for each size of particle the relation between Tyndallmeter reading and concentration. The actual measurements are indicated by the small circles. The crosses for Curve No. I refer to the check run that was made with that suspension. As indicated by the dotted lines, some of the points fall off the plot.

It will be seen at once from this diagram that at a given concentration the smaller the particles the higher is the Tyndallmeter reading. It should be noticed that the curves for the two smallest size of particles bend over at concentrations of about $0.5 \mathrm{~g}$. per liter. This is due to the fact that above this concentration the suspensions absorbed so much light that the linear relation between concentration and intensity of Tyndall beam no longer holds.

Plate II presents curves which have been constructed from Plate I. These curves show the relation between Tyndallmeter reading and the reciprocal of the diameter of the particles, each curve being for one given concentration. These curves show even more clearly that Tyndallmeter

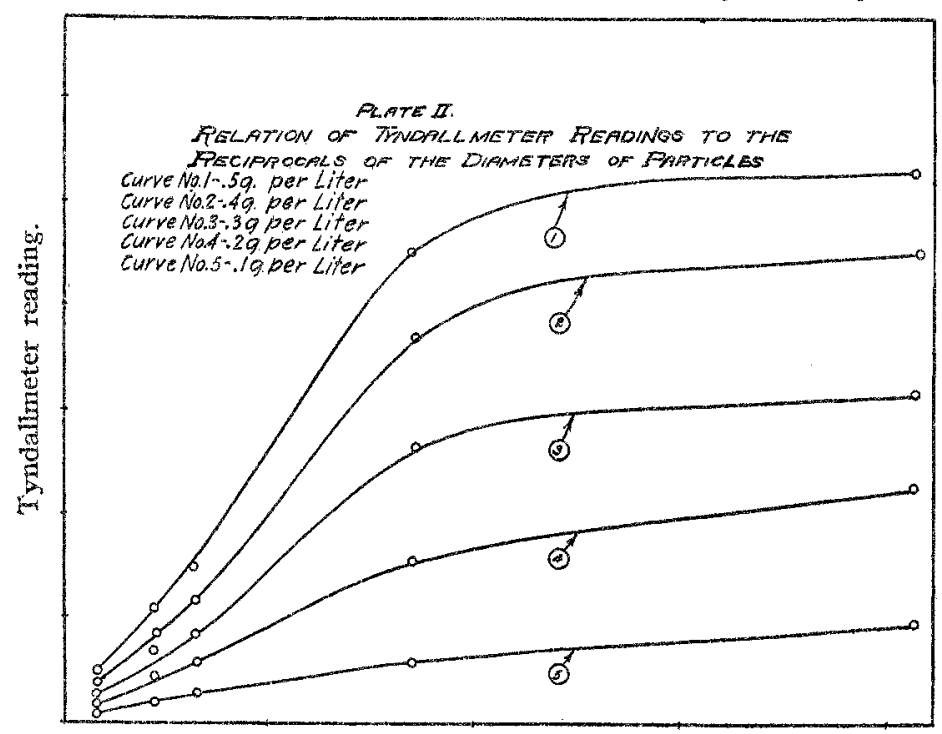

Reciprocal of diameter.

reading increases with increased subdivision. Indeed it will be seen that for the larger particles the Tyndallmeter reading is very nearly proportional to the reciprocal of the particle diameter. This will be referred to later in the discussion of the results.

Plate III shows the same results as those given in Plate I, taking merely the lower concentrations and plotting on a larger scale. With these low concentrations the disturbance due to light absorption by the suspension is avoided. 


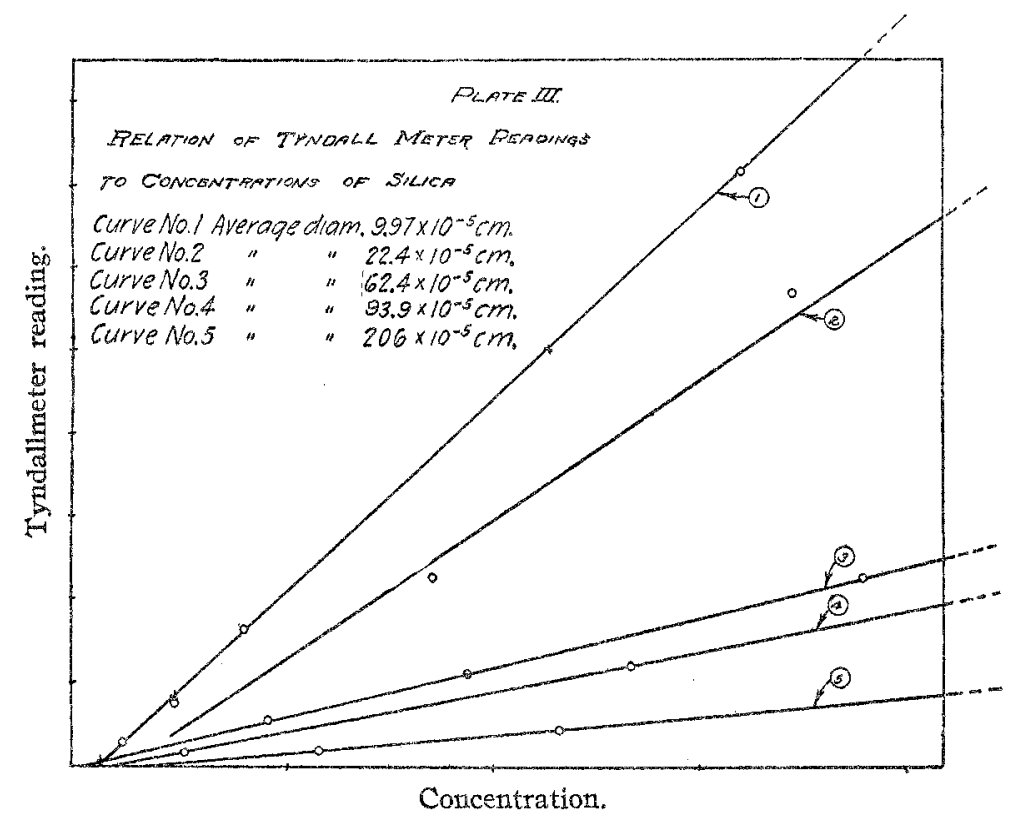

Plate IV for these lower concentrations shows the relation between Tyndallmeter reading and the reciprocal of the diameter. These curves which are for concentration so low that the disturbance produced by light absorption is not present show even more clearly that down to a diameter

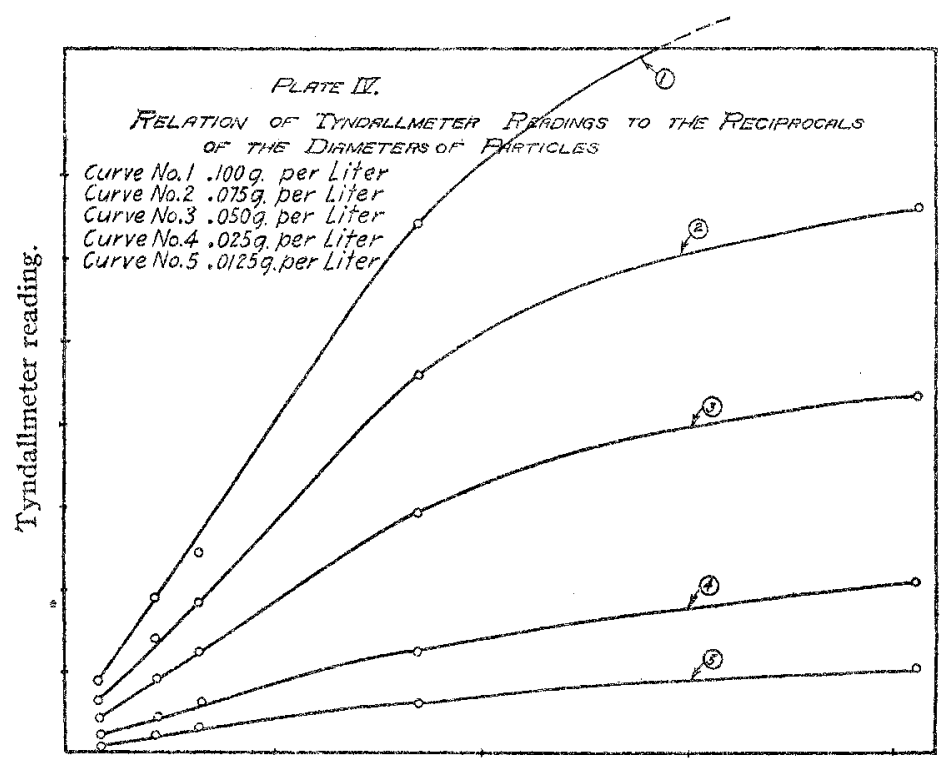

Reciprocal of diameter. 
of about $20 \times 10^{-5} \mathrm{~cm}$. the Tyndallmeter reading is very closely proportional to the reciprocal of the diameter.

Plate $\mathrm{V}$ shows the relation between Tyndallmeter reading and diameter of particle. This plate has less theoretical interest than the ones in which the reciprocal of the diameter are used but is convenient in case the direct relation between Tyndallmeter reading and diameter is desired.

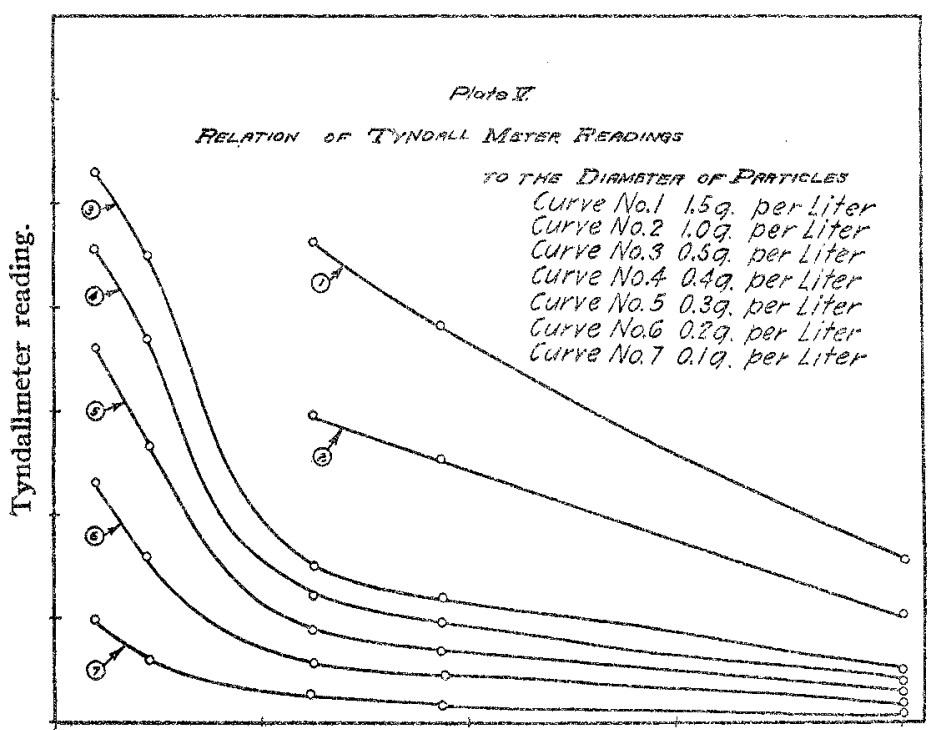

Diameter.

IV. Discussion of Results.

We may now examine the results given by the smokes and silica suspensions to see if they agree with what might be expected. It has been shown by Lord Rayleigh on theoretical grounds that for particles which are small compared with the wave length of light we may expect the intensity of the Tyndall beam to be proportional to the number of particles, $n$, present per cc. and to the sixth power of their diameter, $d^{6}$. For large particles, however, it seems reasonable to expect that the intensity of the Tyndall beam would be proportional to the number of particles per cc., $n$, and to their area of reflecting surface of $d^{2}{ }^{1}$ We can then write as the limiting forms for the relation between Tyndallmeter reading and size of particle

$$
\begin{array}{ll}
T=k n d^{8} & \text { for very small particles } \\
T=k^{1} n d^{2} & \text { for large particles }
\end{array}
$$

where $k$ and $k^{1}$ are constants. Noting that the concentration of the sus-

1 The fact that the intensity of the Tyndall beam would be proportional to the area of surface for large particles was first suggested to us by Mr. P. V. Wells of the Bureau of Standards. 
pension $c$ is proportional to $n d^{3}$, these expressions may be rewritten in the forms

$$
\begin{array}{ll}
T=k c d^{3} & \text { for very small particles } \\
T=k^{1} c / d & \text { for large particles }
\end{array}
$$

Results have already been presented in our article" on "The Disappearance of Smoke in a Confined Space," indicating that for all smokes the Tyndallmeter reading increases with increased degree of subdivision and hence comes nearer following Equation 2 than Equation I. The very satisfactory data presented in the first part of this paper which is merely a sample of work on a large number of materials shows that in general for smokes, increased subdivision results in increased Tyndallmeter reading.

For the silica suspensions the work is even more satisfactory since it was possible to prepare suspensions with a fairly uniform size of particle. It will be seen indeed from the curves, as shown best in Plate IV, that for the four suspensions with the larger particles the Tyndallmeter reading is quite closely proportional to the reciprocal of the diameter of the particles, thus agreeing with Equation 2. The results with the smallest particles show that further subdivision down to a diameter of $9.97 \times 10^{-6}$ $\mathrm{cm}$. still leads to increased Tyndallmeter reading although the rate of increase in the reading is no longer so great as demanded by Equation 2.

This result is also shown by the following table giving the relation between Tyndallmeter reading $T$ and diameter of particle $d$ for a given concentration ( $0.3 \mathrm{~g}$. per liter) of silica suspension:

$\begin{array}{cr}\text { d. Cm. } \times 10^{-8} . & \text { TABLE IV. } \\ 9.97 & T . \\ 22.4 & 175 \\ 62.4 & 134 \\ 93.9 & 44 \\ 206.0 & 35 \\ & 14\end{array}$

The only quantitative results in this field of which we know are those of Mecklenburg, ${ }^{1}$ which were obtained with sulfur suspensions. The following table is based on his results, and gives the relation between Tyndallmeter reading $T$ and diameter of particle $d$ for a given concentration of sulfur suspension.

$\begin{array}{cc}\text { d. Cm. } \times 10^{-8} . & \text { Tabit V. } \\ 0.05 & T . \\ 0.10 & 0.05 \\ 0.20 & 0.88 \\ 0.30 & 6.00 \\ 0.42 & 13.60 \\ 0.93 & 145.5 \\ 2.46 & 180.0 \\ 8.40 & 190.0 \\ & 383.0\end{array}$

${ }^{1}$ Loc. cit. 
Mecklenburg's results show in accordance with Lord Rayleigh's law that for very small particles the Tyndallmeter reading increases proportionally to the cube of the particle diameter. They also show, however, that at a diameter of $8.40 \times \mathrm{ro}^{-5} \mathrm{~cm}$. the Tyndallmeter reading is still increasing with size of sulfur particle while our results which begin where his leave off show that at this size of silica particle the Tyndallmeter readings are decreasing with increasing diameter. This discrepancy is further emphasized by our results with smokes which, although only qualitative in nature, nevertheless show definitely that even for much smaller particles Tyndallmeter reading decreases at a given concentration with size of particles.

Further investigations on dispersoids, bridging the gap between the region where Lord Rayleigh's law holds and the region where Tyndallmeter reading is proportional to total reflecting surface would be desirable.

WAGHINGTON, D. C.

\section{AN ELECTRICAL PRECIPITATOR FOR ANALYZING SMOKES.}

By Richard C. Torman, L. H. Reyerson, A. P. Brooks and H. D. SMrth. Received January 17, 1919.

The following note presents a description of an electrical precipitating apparatus for determining the concentration of smokes. The apparatus was developed by the Dispersoid Section, Research Division, Chemical Warfare Service. ${ }^{1}$

The purpose of the work was to obtain a general form of apparatus which could be used for the analysis of any smoke independent of its chemical nature. The apparatus consists essentially of a modified Cottreil precipitator, with a central wire as cathode surrounded by a cylindrical foil as anode. The smoke to be analyzed is drawn through the apparatus at a known rate and the particles of smoke precipitated on the foil by means of a high voltage, direct current. The determination of concentration is made by measuring the volume of air that passes through the apparatus and weighing the foil before and after precipitation.

The most important consideration to be kept in mind in designing such an apparatus is to make the foil very light, so that the precipitated material shall form as large a fraction as possible of the total weight. For this reason we used aluminum foil o.oor inch in thickness. Thin tin foil was tried but was easily torn in handling and did not have spring enough to keep it cylindrical in shape. This latter point is very important, since a distortion of the cylindrical form leads to sparking instead of the desired corona discharge.

A second consideration to keep in mind, in the design of the apparatus,

1 The publication of this note has been authorized by Major-General William $\mathfrak{I}$. Sibert, Chief of Chemical Warfare Service, U. S. A. 